\title{
LXV. Considerations on the two memoirs of Sir B. C. Brodie on the calculus of chemical operations
}

\section{M.A. Naquet}

To cite this article: M.A. Naquet (1879) LXV. Considerations on the two memoirs of Sir B. C. Brodie on the calculus of chemical operations, Philosophical Magazine Series 5, 7:45, 418-432, DOI: $10.1080 / 14786447908639636$

To link to this article: http://dx.doi.org/10.1080/14786447908639636

曲 Published online: 13 May 2009.

Submit your article to this journal $\sqsubset \pi$

Џ Article views: 3

Q View related articles $\square$ 


\section{[ 418 ]}

LXV. Considerations on the two Memoirs of Sir B. C. Brodie on the Calculus of Chemical Operations. By M. A. NAQUET*.

TT is not our intention here to express a complete judgment 1 upon the work of Sir B. C. Brodie. This work comprises a mathematical part, of which we are not competent to judge, and a chemical part, upon which we have the right of pronouncing. It is with the latter alone that we mean to occupy ourselves here.

We may begin by saying that whatever may be the final judgment pronounced upon the work of Sir B. C. Brodie, that work appears to us remarkable; and it is that which has induced us to make it known to the French public.

The application of Algebra to the experimental sciences, the substitution of "theories," based upon facts and demonstrated laws, for "systems" which only rest upon metaphysical hypotheses, is the end towards which science ought to tend; and if systems are necessary for the arrangement of phenomena, and for the discovery of new phenomena in those points where the progress of science has not yet allowed them to be replaced by theories, this substitution ought, nevertheless, to be effected as soon as practicable.

We will say at once that Sir B. Brodie is unjust in denying the discoveries which are due to the atomic notation. It is by means of this notation and the probabilities deduced by it that a number of syntheses have been effected-such, for example, as the synthesis of the phenols, those of the acids of the salicylic series, of secondary and tertiary ammonia compounds, etc. etc. How could it, in fact, be otherwise? As M. Dumas says, in his 'Leçons de philosophie chimique,' a bypothesis created for the explanation of twenty phenomena, to which it is adequate, is necessarily applicable to ten, twenty, thirty other unknown phenomena on the track of which it places the observer. But, while fully recognizing the superiority of a "theory" over a system, we refuse to abandon our system unless the theory be complete enough to render all the services which the system has rendered. It does not appear to us that at present the notation of Sir B. Brodie has gone so far as to be able completely to replace the existing notation; but I do not consider these reasons sufficient to condemn it.

When Gerhardt modified the notation in use before his

* M. A. Naquet has translated into French the two memoirs referred to, on which translations the critical observations of $\mathbf{M}$. A. Naquet are based. These have been translated into English under my supervision.B. C. B. (Moniteur Scientifique du Docteur Quesneville, Nov. 1878, March and April 1879.) 
time, this notation did not assume at first the form it has since acquired : it has been perfected, modified, almost transformed; it is, however, from Gerhardt that it dates, and to him the honour of it is due. Similarly Sir B. C. Brodie makes a bold attempt on a new path which may lead to great results; and we must beware of rejecting what he brings on the ground that it is incomplete. The mathematical analysis proposed by him is as yet but a germ; worked out and developed, it may become an organism.

This said, and all reservations made as much in favour of as against the new method, we may say at once that the existing atomic notation may be divided into two parts :- that which is entirely hypothetical and metaphysical, and which explains phenomena by the grouping of atoms ; and that which, in spite of the words atom and molecule which offend Sir B. C. Brodie, is not more hypothetical than the notation of Sir B. C. Brodie himself. The whole is, in fact, a matter of definition. How do we define a molecule? It is the smallest portion of matter at which we can arrive by physical division, and of which the weight is equal to two volumes of the vapour considered in relation to the weight of one volume of hydrogen, both at the normal conditions of temperature and pressure. How do we define an atom? It is the smallest quantity of a given portion of matter attainable by chemical division, which is not subdivided in any operation, and which is always transported integrally from one combination to another. A molecule, then, setting aside all metaphysical senses, is the weight of two volumes of a gas or a vapour; an atom is the weight of two, of one, or of half a volume of a gas or a vapour, according as, in chemical reactions, the molecule is transported intact or is subdivided into smaller weights.

Now what does a " simple weight" represent for Sir B. C. Brodie? It is a weight which in being transported from one combination to another is not "distributed." What does a compound weight represent to him? It is a weight which in the course of chemical operations is divided, is "distributed." Finally, what does he term the "unit of ponderable matter"? The weight of one volume, say of 1000 cub. centims., of a gas or of a given vapour.

Let us suppose that Sir B. C. Brodie had accepted his hypothesis $\alpha^{2}$ instead of stopping at the hypothesis $\alpha$. All his units would then become equal to our molecules; those of his "units" which were not "distributed" would have been identical with those of our molecules (mercury, for instance) which we consider as composed of a single atom. Those of his " units" which were "distributed" would have been 
identical with those of our molecules which we consider as composed of several atoms. It is, in fact, absolutely identical to take $\mathrm{H}=1$ and to bring all the molecules to two volumes, or to bring all the molecules to one volume and to take $\mathrm{H}=\frac{1}{2}$. The numbers obtained would be the same in the two cases.

Now, if Sir B. C. Brodie has been induced, by the serious considerations given in his memoir, to prefer the hypothesis $\alpha$ to the hypothesis $\alpha^{2}$, he does not, however, consider the hypothesis $\alpha^{2}$ as more metaphysical, less scientific than the other; and if it had not been his object to explain the "law of even numbers," he would have adopted it*.

With the hypothesis $\alpha^{2}$, there would have been nothing different between our notation and that of Sir B. C. Brodie, nothing except the substitution of the words "units," "distributed weights," "simple weights," for the words "molecules," "molecules composed of several atoms," and "atoms." Now, when it is well understood that no importance, no metaphysical signification, is attached to the words "molecules" and atoms," but that the expressions are simply taken as indicative of facts previously announced, the question of words signifies nothing, and it matters little whether the same ideas are expressed by " molecules" and "atoms," or by " units," "simple or undistributed weights," and "compound or distributed weights" $\dagger$. It matters no more than it matters whether a given thought be expressed in one language or in another, provided that the expression be clear and unequivocal.

Let us go farther. Sir B. C. Brodie recognizes that certain "weights" which are regarded absolutely (that is, when the whole of the chemical system is considered) as " compound or distributed," cannot in a limited system of operations be distributed, and act like "simple weights." These are "simple relative weights." Now, when we give the name of "com-

* The fact is that; but for the law of even numbers, the system a could not be constructed at all.-B. C. B.

$\dagger$ All this is perfectly true; but it is eminently undesirable to express different ideas by the same term, which leads to confusion. The ideas expressed by the terms "simple weights" and "undistributed weights," are not the ideas expressed by the terms "atoms" and "molecules," but rather those ideas divested of what M. Naquet terms their "metaphysical signification," which are not the same thing. Indeed the adrocates of the atomic basis of Chemistry would not, I imagine, be very well pleased with M. Naquet's description of an atom as "a metaphysical entity."

The identity of system $\alpha^{2}$ with our present system is limited to the identity of the notation by which the units of matter are expressed. The Algebraical method of working with these symbols as developed in Part II., which is an essential feature of this Calculus and peculiar to it, is not found in our present system.-B. C. B. 
pound radical" to the portion of matter represented by $\mathrm{C}_{2} \mathrm{H}_{4}$ or $\mathrm{C}_{2} \mathrm{H}_{5}$, for instance, we do not express any other idea. The compound radical represents to us a portion of matter which in the series of phenomena under consideration is transported integrally from one combination into another, although it may, in other circumstances, be decomposed into simpler elements. "Compound radical," or " simple relative weight," are therefore one and the same thing. Here, again, is a matter of words.

We may add that the substitution of hypcthesis $\alpha$ for hypothesis $\alpha^{2}$ is not fundamental. If, supposing that Sir B.C.Brodie had not written his two memoirs, it had been shown that the bodies, so called, of uneven atomicity contain hydrogen, and correspond to the general formula $\mathrm{HR}_{2}$ or $\mathrm{H}_{2} \mathrm{R}_{4}$, immediately our existing notation would be transformed, except for the use of Greek letters, into that of Sir B. C. Brodie, without any alteration in the general system, unless it were the important discovery of the compound nature of a series of bodies hitherto considered as elementary.

To set against this there is in the existing notation a series of hypothetical considerations for the expression of fine isomers, which Sir B. C. Brodie rejects. Thus, when we express the isomeric relations of aldehyde, acetylenic alcohol, and oxide of ethylene by the formulæ
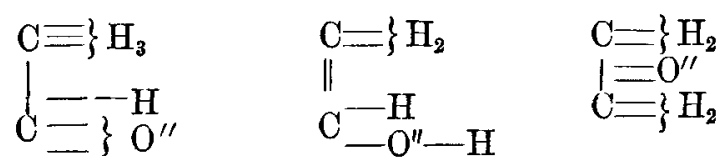

we make a hypothesis on the mode of grouping these atoms considered as metaphysical entities.

It is the same when we try to account for the differences existing between isomers such as salicylic, oxybenzicic, and paraoxybenzoic acids, and explain them by the place occupied in relation to each other by the groups $\mathrm{OH}$ and $\mathrm{CO}_{2} \mathrm{H}$ and the benzol-group $\mathrm{C}_{6} \mathrm{H}_{6}$.

We will return presently to this hypothetical part of our notation. Let us pause for a moment on the part common to the existing notation and the notation of Sir B. C. Brodie, and see if, in fact, the hypothesis $\alpha$, setting aside the difficulty caused by the necessity of considering as compounds a mass of simple weights, offers the advantages over the hypothesis $\alpha^{2}$ which Sir B. C. Brodie asserts. According to him, a hypothesis is acceptable when it accounts for all the known facts. Of two hypotheses which equally account for all the facts, that 
one ought to be preferred which is the more limitative. In other words, if a hypothesis A accounts for all the facts and does not suggest any improbable phenomenon, while another hypothesis $A_{1}$ accounting equally for all the known facts suggests a considerable number of unknown and improbable facts, it is hypothesis A which we ought to select.

We fully accept these principles; but let us see their conclusions. The hypothesis $\alpha^{2}$, Sir B. C. Brodie says, permits the regular explanation, by means of integral positive factors, of the symbols of all the known facts. But it admits besides of the similar construction of symbols of an equal number of substances which the law of even numbers rejects as impossible to realize. The hypothesis $\alpha$, on the contrary, while permitting the expression by symbols, by means of integral positive factors, of known facts, excludes the possibility of representing in the same way, by means of symbolic expressions, the substances which do not obey the law of even numbers. The hypothesis $\alpha$ is therefore superior to the hypothesis $\alpha^{2}$.

Such is the argument of Sir B. C. Brodie; and we should consider it irreproachable if bypothesis a were not, though more limitative on one side, more extensive on the other, and if, from this point of view, what is gained in one sense were not lost in an opposite sense.

But, first, the hypothesis $\alpha$ compels us to admit that chlorine, bromine, iodine, nitrogen, phosphorus, arsenic, antimony, bismuth, potassium, sodium, etc., are compound bodies corresponding to the general formula $\mathrm{HR}_{2}$ or $\mathrm{H}_{2} \mathrm{R}_{4}$. This is a serious obstacle against accepting this hypothesis; for though there may be nothing impossible in this supposition, neither is there anything demonstrated: we anticipate experience, and thus we enter upon a path which threatens to lead us far.

This is not all. The existing theory, which considers chlorine, bromine, iodine, nitrogen, etc. as elementary bodies, leads us to consider as products of "substitution" the compounds resulting from the action of chlorine, bromine, or iodine on organic hydrogenized bodies, as well as the compound ammonias produced by the action of simple ethers on ammonia. The number of these products is thus limited.

Let us take, for example, the action of chlorine on marshgas, $\mathrm{CH}_{4}$. Theory indicates that this action ought to produce four bodies, and four bodies only, as indicated in the the following equations :-

$$
\begin{aligned}
& \mathrm{CH}_{4}+\mathrm{Cl}_{2}=\mathrm{HCl}+\mathrm{CH}_{3} \mathrm{Cl}, \\
& \mathrm{CH}_{3} \mathrm{Cl}+\mathrm{Cl}_{2}=\mathrm{HCl}+\mathrm{CH}_{2} \mathrm{Cl}_{2}, \\
& \mathrm{CH}_{2} \mathrm{Cl}_{2}+\mathrm{Cl}_{2}=\mathrm{HCl}+\mathrm{CH} \mathrm{Cl}_{3}, \\
& \mathrm{CH} \mathrm{Cl}_{3}+\mathrm{Cl}_{2}=\mathrm{HCl}+\mathrm{CCl}_{4} .
\end{aligned}
$$


And, in fact, the action of chlorine on marsh-gas produces four products of substitution-chloride of methyl $\mathrm{CH}_{3} \mathrm{Cl}$; chloruretted chloride of methyl, $\mathrm{CH}_{2} \mathrm{Cl}_{2}$; chloroform, $\mathrm{CH} \mathrm{Cl}_{3}$; perchloride of carbon, $\mathrm{C} \mathrm{Cl}_{4}$-and four only.

Similarly, ammonia having as its formula $\mathrm{NH}_{3}$, and chloride of ammonium $\mathrm{NH}_{4} \mathrm{Cl}$, we ought to be able to substitute three alcoholic radicals for the hydrogen of ammonia, and four of these radicals for the hydrogen of ammonium contained in the ammonia chloride, whence result three compound free ammonias and three only, four compound ammoniums in the state of combination and four only, as indicated by the following equations :-

$$
\mathrm{NH}_{3}+\mathrm{C}_{2} \mathrm{H}_{5} \mathrm{I}=\mathrm{HI}+\mathrm{NH}_{2}\left(\mathrm{C}_{2} \mathrm{H}_{5}\right)=\text { ethylamine; }
$$

$\mathrm{NH}_{2}\left(\mathrm{C}_{2} \mathrm{H}_{5}\right)+\mathrm{C}_{2} \mathrm{H}_{5} \mathrm{I}=\mathrm{HI}+\mathrm{NH}\left(\mathrm{C}_{2} \mathrm{H}_{5}\right)_{2}=$ diethylamine; $\mathrm{NH}\left(\mathrm{C}_{2} \mathrm{H}_{5}\right)_{2}+\mathrm{C}_{2} \mathrm{H}_{5} \mathrm{I}=\mathrm{HI}+\mathrm{N}\left(\mathrm{C}_{2} \mathrm{H}_{5}\right)_{3}=$ triethylamine; $\mathrm{N}\left(\mathrm{C}_{2} \mathrm{H}_{5}\right)_{3}+\mathrm{C}_{2} \mathrm{H}_{5} \mathrm{I}=\mathrm{N}\left(\mathrm{C}_{2} \mathrm{H}_{5}\right)_{4} \mathrm{I}=$ iodide of triethylammonium.

And this is, in fact, what occurs : each compound monatomic alcohol-radical produces three compound free ammonias and one quaternary ammonium, and these four ammonia derivatives only. Finally, from the relations which exist between the derivatives of the chlorine substitution of hydrocarbons and alcohols, the latter also are considered as products of substitution resulting from the exchange of $\mathrm{H}, \mathrm{Cl}, \mathrm{Br}$, or $\mathrm{I}$ for the group $\mathrm{OH}$. From the relations which connect alcohols to aldehydes, aldehydes to acids, acids to amides, the number of alcohols, acids, aldehydes, and amides is limited, like that of the chlorine derivatives, by the number of chlorine derivatives contained in each hydrocarbon.

It is not the same with Sir B. C. Brodie's notation. Marshgas, for example, being $\alpha^{2} \kappa\left(=\mathrm{CH}_{2}, \mathrm{C}=6\right)$, and chlorine being $\alpha \chi^{2}$, the reaction of chlorine on the hydrocarbon becomes

$$
\underbrace{\alpha^{2} \kappa}_{\text {Marsh-gas. }}+\underbrace{\alpha \chi^{2}}_{\text {Chlorine. }}=\underbrace{\alpha \chi}_{\begin{array}{c}
\text { Chlorhydric } \\
\text { acid. }
\end{array}}+\underbrace{\alpha^{2} \kappa \chi}_{\begin{array}{c}
\text { Chloride } \\
\text { of methyl. }
\end{array}}
$$

That is to say, the chloride of methyl, which in our notation corresponds to the formula $\mathrm{CH}_{3} \mathrm{Cl}$, and represents a product of substitution of chlorine for hydrogen, in Sir B. C. Brodie's notation represents a simple addition of the prime factors of chlorine $\chi$ to marsh-gas. Similarly, methylic alcohol, $\mathrm{CH}_{3}, \mathrm{OH}$, which in our notation is a product of substitution, becomes in the new notation $\alpha^{2} \kappa \xi$-that is to say, a simple product of addition. 
It is the same for the compound ammonias. The equations by which we have represented the formation of these bodies take, in Sir B. C. Brodie's notation, the following form :-

$$
\underbrace{\alpha^{3} \kappa^{2} \omega}_{\begin{array}{c}
\text { Iodide of } \\
\text { ethyl. }
\end{array}}+\underbrace{\alpha^{2} \nu}_{\text {Ammonia. }}=\underbrace{\alpha \omega}_{\begin{array}{c}
\text { Iodhydric } \\
\text { acid. }
\end{array}}+\underbrace{\alpha^{4} \kappa^{2} \nu .}_{\text {Ethylamine. }}
$$

$\alpha^{3} \kappa^{2} \omega+\alpha^{4} \kappa^{2} \nu=\alpha \omega+\alpha^{6} \kappa^{4} \nu=$ diethylamine, $\alpha^{3} \kappa^{2} \omega+\alpha^{6} \kappa^{4} \nu=\alpha \omega+\alpha^{8} \kappa^{6} \nu=$ triethylamine, $\alpha^{3} \kappa^{2} \omega+\alpha^{8} \kappa^{6} \nu=\alpha^{11} \kappa^{8} \omega \nu=$ iodideof triethylammonium. That is to say, the ammonias and the compound ethylic salts of ammonium result simply from the addition of ethylene, $\alpha^{2} \kappa^{2}$ to ammonia, or to the salts of ammonium. We have, in fact,

$$
\underbrace{\alpha^{2} \nu}+\underbrace{\alpha^{2} \kappa^{2}}=\alpha^{4} \kappa^{2} \nu=\text { ethylamine; }
$$

Ammonia. Ethylene.

$$
\underbrace{\alpha^{4} \kappa^{2} y}+\underbrace{\alpha^{2} \kappa^{2}}=\alpha^{6} \kappa^{4} y=\text { diethylamine; }
$$

Ethylamine. Ethylene.

$$
\alpha^{6} \kappa^{4} \nu+\alpha^{2} \kappa^{2}=\alpha^{8} \kappa^{6} \nu=\text { triethylamine; }
$$

Diethyl-

amine.

$$
\underbrace{\alpha^{3} \omega \nu}+\left(\alpha^{2} \kappa^{2}\right)^{4}=\alpha^{11} \kappa^{8} \omega \nu=\text { iodide of tetrethylammonium. }
$$

Iodide of ammonium.

Now, since Sir B. C. Brodie rejects every atomic speculation which would necessarily place a limit to these different additions, his theory shows for each hydrocarbon an indefinite number of chlorine, bromine, or iodine derivatives, and an equally indefinite number of alcohols, aldehydes, and acids; it shows also an indefinite number of compound ammonias for each alcohol, of amides for each acid, \&c. \&c.

We can, in fact, according to the new system of notation, perform upon the symbol of perchloride of carbon, $\alpha^{2} \kappa \chi^{4}$, the same operation that we can perform upon marsh-gas, perchloride of methyl, chloruretted chloride of methyl, and chloroform themselves. Thence we obtain the following equations:-

$$
\underbrace{\alpha^{2} \kappa \chi^{3}}+\underbrace{\alpha \chi^{2}}=\underbrace{\alpha \chi}+\underbrace{\alpha^{2} \kappa \chi^{4}},
$$

Chloroform. Chlorine. Chlorhydrio Perchloride

$$
\underbrace{\alpha^{2} \kappa \chi^{4}}_{\begin{array}{c}
\text { Porchloride } \\
\text { of carbon. }
\end{array}}+\underbrace{\alpha \chi^{2}}_{\text {Chlorine. }}=\underbrace{\alpha \chi}_{\begin{array}{c}
\text { Chlorhydric } \\
\text { acid. }
\end{array}}+\alpha^{2} \pi \chi^{5} \text { ? }
$$


It is absolutely impossible to express this last compound in our existing notation.

Similarly, tetratomic alcohol, which would have for its formula $\mathrm{C}(\mathrm{OH})_{4}$, an alcohol which is unknown, but of which the derivatives are known, ought to be written, according to Sir B. C. Brodie, $\alpha^{2} \xi^{4}$. But Sir B. C. Brodie's theory shows equally an alcohol $x^{2} \kappa \xi^{5}$, which our existing notation rejects as impossible.

Finally, besides the triethylamine, $\left.\left(\alpha^{2} \kappa^{2}\right)^{3}\right) \alpha^{2} v$, and the iodide of tetrethyl-ammonium $\left(\alpha^{2} \kappa^{2}\right)^{4}\left(\alpha^{2} v\right)(\alpha \omega)$, the notation of Sir B. C. Brodie shows the compound ammonias $\left(\alpha^{2} \kappa^{2}\right)^{n} \alpha^{2} v$ and the compound iodides of ammonium, $\left(\alpha^{2} \kappa^{2}\right)^{n+1}\left(\alpha^{2} v\right)(\alpha \omega)$. These last bodies, unlike the preceding, might be written in our existing notation, which would permit the expression of these compounds by the formula

$$
\left(\mathrm{C}_{2} \mathrm{H}_{4}\right)^{n} \mathrm{NH}_{3} \text { and }\left(\mathrm{C}_{2} \mathrm{H}_{4}\right)^{n+1} \mathrm{NA}_{4} \mathrm{Cl} \text {; }
$$

but our notation allows us also to consider them as compounds of substitution, instead of considering them as compounds of addition, which limits their number.

Now, up to the present time there has never been obtained a number of chlorine, bromine, or iodine derivatives of a hydrocarbon greater than the number of the atoms of hydrogen which this hydrocarbon contains. There has never been obtained a number of alcohols greater than that of the chlorine, bromine, or iodine derivatives. Finally, there has never been obtained for each monatomic alcohol a number of compound anmonias greater than three, and a number of compound salts of ammonium greater than four.

On the other hand, the law of even numbers, which Sir B. C. Brodie has so much at heart, is far from being demonstrated, since the exception of the oxides of nitrogen cannot be eliminated unless we admit the dissociation of our element nitrogen. But even if the law of even numbers were absolutely demonstrated, it would still not prove that a body which cannot exist in a free state may not exist in a state of combination. And, finally, if this explanation itself be inadmissible, since the notation $a$ of $\operatorname{Sir}$ B. C. Brodie shows an innumerable multitude of improbable bodies, and he does not point out to us any rule for eliminating them, which is contrary to that which actually occurs in the existing notation (and to that which occurs in the notation of Sir B. C. Brodie), for the bodies which do not satisfy the law of even numbers, the hypothesis $\alpha$ appears to us in all points inferior in the existing state of science to the hypothesis $\alpha^{2}$.

Now the hypothesis $a^{2}$ is nothing else than our existing 
notation without the speculations explanatory of the fine isomers mentioned above. Are these speculations, which could be perfectly well rejected even with our existing notation, out of date and already useless? I do not think so. They may become useless one day. We shall perhaps find in thermochemistry the means of explaining these fine isomers, and applying to them mathematical expressions. But until then these speculations appear to us useful, because they arrange facts which would otherwise remain without arrangement; and they correspond well enough to the actual phenomena to allow us to determine, notably in the aromatic series, the number of these fine isomers possible for each term of the series.

Even if we do not take into consideration the extreme difficulty that there is in replacing one notation by another, and the momentary confusion introduced by it into science, a confusion which ought not to be permitted unless the change offers an undeniable advantage, even if we do not take into consideration this difficulty, we should oppose the immediate adoption of Sir B. C. Brodie's notation, because it seems to us to open the door to a crowd of suppositions more considerable than those it aims at eliminating, and because, from the point of view of the arrangement and anticipation of facts, it does not provide us with the means of dispensing with those atomic speculations which, without being intimately connected with the existing notation, may nevertheless be joined to it, and cannot in any case be adapted to the notation of $\operatorname{Sir} B$. C. Brodie.

Are we then to conclude that Sir B. C. Brodie's work has no value? Far from us indeed be such a thought. If we had been of this opinion, we should not have taken the trouble to translate it. It opens out a new method which, enlarged and perfected, will permit the application of Algebra to Chemistry, and the substitution for our "system of chemistry" of a true "theory of chemical events." Even if this result be not produced, if the imperfections with which we charge the new method of notation continue to exist, if there only remain of the notation of Sir B. C. Brodie his hypothesis $\alpha$, including that of the compound constitution of chlorine, nitrogen, potassium, and their compounds, the work would still be useful.

As long as we have to do with a "system," a hypothesis with no other object than that of arranging known facts and discovering new facts, two different hypotheses may legitimately be employed, provided that they answer to the requisite conditions. It is thus that the hypotheses of emission and undulations have long been tacitly accepted in Optics. It 
might happen in this case also that two different hypotheses, both including the whole of the known phenomena, should lead to different deductions, and present different consequences which should guide the operator into two distinct paths. When things occur thus, it is useful to accept the two hypotheses at once, and since no objective reality is attributed to them until experience has decided (when experience can decide), both are undeniably useful. Sir B. C. Brodie's system, by showing the possible decomposition of the whole of one class of our elements, and by indicating one of the probable cases in which these elements are decomposed (the cases of binoxide and tetroxide of nitrogen), renders a real rervice to chemical science, and deserves to be known and studied. It will deserve this still more, if it be found that in working it out and perfecting it its defective sides are caused to disappear and its completion effected. It will help to place Chemistry on a solid foundation; and such an attempt has a right to the sympathetic attention of the whole scientific world.

Postscriptum. Since these lines were written some curious observations have allowed scientific men to consider as possible, as even probable, the production of free hydrogen by the action of an extremely elevated temperature on the greater number of our elementary bodies. It is useless to demonstrate -it demonstrates itself-the value which this discovery, if it is confirmed, would give to Sir B. C. Brodie's hypothesis.

Note on an Objection made by M. Naquet in lis preceding "Observations." By Sir B. C. BRoDIE, F.R.S.*

M. Naquet has had the kindness to forward to me the proofs of his "Considerations" on my Memoirs. It is not my wish to comment here on his remarks; but there is one point in reference to which the remarks of $M$. Naquet are founded on a pure misapprehension of the state of the case. This point is of fundamental importance; and I will give a few words of explanation in regard to it. Indeed, if the difficulties which he has created really existed, the atomic method would have in some respects a great advantage over the method of this Calculus. I will explain the matter as briefly as possible. The objection of M. Naquet is this. We may, according to the atomic method, operating by way of substitution of atom for atom, foresee in certain cases the number of practicable substitutions. Thus, marsh-gas $\left(\mathrm{CH}_{4}\right)$ containing four atoms of hydrogen, we may substitute in it chlorine for hydrogen

* From the Moniteur Scientifque of Dr. Quesneville, April 1879. 
four times and no more. Similarly in ammonia $\left(\mathrm{NH}_{3}\right)$ three analogous substitutions are possible. Whence we have, for example, the three ammonia bases, methylamine, dimethylamine, trimethylamine. Again, in chloride of ammonium $\left(\mathrm{NH}_{4} \mathrm{Cl}\right)$ we may have four such substitutions; and extending this principle, we are able to anticipate in numerous cases the precise number of similar derivatives. All this, it is asserted, is the work of the atomic method. Now the theory before us, says M. Naquet, does nothing of the kind, and, indeed, is incapable of doing it. We have $\alpha^{2} \kappa \chi^{3}$ as the symbol of chloroform, and $\alpha^{2} \kappa \chi^{4}$ as the tetrachloride of carbon. All that is here done to form the chlorine derivatives of marsh-gas is to add the weight $\chi$ over and over again to the weight $a^{2} \kappa$. Go on in the same direction, the next step brings you to $\alpha^{2} \kappa \chi^{5}$, an utterly impossible, or at least an eminently improbable, entity, which yet is not excluded from the system.

The reply to this is that M. Naquet does not give a correct account of the process by which these derivatives are constructed. We cannot make $\alpha^{2} \kappa \chi^{5}$ by the same process as that by which $\alpha^{2} \kappa \chi^{4}$ is manufactured. After $\alpha^{2} \kappa \chi^{4}$ is placed an insurmountable barrier to progress in this direction which he does not see. He can go so far, but no further.

M. Naquet's criticism is based on a distinction for which he certainly is not responsible, as it is made in every chemical treatise, which is necessitated by the material mode of treatment of the atomic theory, but which, in this Calculus, does not exist, namely the distinetion between Addition and Substitution of atoms. For us the two processes are merged in one. (Part II. Section III. (9).)

Let us consider the equation which expresses the relation between the chloride of iodine and its constituents,

or

$$
\alpha \chi^{2}+\alpha \omega^{2}=2 \alpha \chi \omega,
$$

$$
\alpha \chi^{2}+\alpha \omega^{2}-2 \alpha \chi \omega=0 \text {. }
$$
thus

This equation vanishes when $\chi=\omega$. It may be written

$$
\alpha(\chi-\omega)(\chi-\omega)=0 .
$$

In this event $\alpha$ is constant, and it occurs in two ways by the substitution of $\omega$ for $\chi$, which substitution is expressed by the symbol $(\chi-\omega)$, and we have:-

Symbol of the unit of chlorine............. $\alpha \chi$,

$" \quad " \quad$ chloride of iodine... $\quad$ iodine .............. $\alpha \omega \omega$,

in which symbols the relation of substitution connecting these units is apparent. 
Now taking the equation

we have similarly

$$
\alpha+\alpha \chi^{2}=\alpha 2 \chi
$$

$$
\alpha(\chi-1)(\chi-1)=0
$$

an event in which $\alpha$ is constant, and which occurs in two ways by the substitution of 1 for $\chi$ - that is, by the operation $(\chi-1)$. Reasoning as before, we have:-

$$
\text { Symbol of the unit of chlorine }
$$

$" \quad " \quad$ hydrochloric acid... $"$. $\quad$ hydrogen...........

Now the symbol 1 which appears in the symbols of hydrochloric acid and of hydrogen is not the symbol of any real weight, but is the symbol of an empty unit of space serving to mark the place where matter is not, but has been, and may be again. The explicit introduction of this symbol into the symbol of the unit of hydrogen limits the number of the operations $\chi$ (or of any operation which may be substituted for $\chi$ ) which may be performed upon $\alpha$, the unit of hydrogen, to two. Thus the operations $\omega$ or $\beta$ (which may be substituted for $\chi$ ) may be performed once and twice upon the unit of hydrogen, but no more. Hence we have:-

$$
\begin{array}{ccc}
\text { Symbol of the unit of hydrogen............ } & \alpha 11, \\
", & \text { hydriodic acid...... } & \alpha \omega 1, \\
" & \text { iodine .............. } & \alpha \omega \omega ;
\end{array}
$$

but we have no means of making $\alpha \omega^{3}$.

Now in the symbol $a^{2} \kappa$, the symbol of the two combined units of hydrogen, which (regarded purely as the symbol of the weight of the combined hydrogen) is $\alpha^{2}$, becomes, by the explicit introduction of the symbol $1, \alpha^{2}(1,1,1,1)$, and the symbol of marsh-gas is $\alpha^{2} \kappa(1,1,1,1)$. In this case four successive substitutions of $\chi$ for 1 are possible, by which the following units are constructed:-

$$
\begin{aligned}
& \alpha^{2} \kappa(\chi, 1,1,1), \\
& \alpha^{2} \kappa(\chi, \chi, 1,1), \\
& \alpha^{2} \kappa(\chi, \chi, \chi, 1), \\
& \alpha^{2} \kappa(\chi, \chi, \chi, \chi),
\end{aligned}
$$

which are the symbols of the four chlorine derivatives of marsh-gas. But we have no means of constructing the unit $\alpha^{2} \kappa \chi^{5}$. (Part II. Section III. (12).)

Again, the unit of nitrogen $\alpha \nu^{2}$ is derived from the unit of hydrogen by the substitution of $v$ for 1 in that unit; and we have:-

$$
\begin{aligned}
& \alpha(1,1) \text { hydrogen, } \\
& \alpha(v, 1) \text { (unknown), } \\
& \alpha(v, v) \text { nitrogen. }
\end{aligned}
$$

Phil. Mag. S. 5. Vol. 7. No. 45. June 1879. 
Effecting this substitution not in one but in two combined units of hydrogen, we have:-

Symbol of two combined units of hydrogen $a^{2}(1,1,1,1)$,

Symbol of the unit of ammonia ............. $a^{2}(v, 1,1,1)$.

It hence appears that we can effect in the unit of ammonia three substitutions of $\chi$ (or of any value of $\chi$ ) for 1 , but no more. Now $\alpha \kappa$ is the symbol of such a value of $\chi$, a fact ascertained by repeated experiments; and the symbols of these three derivatives are:-

hence the equation

$$
\begin{aligned}
& \alpha^{2} \nu(\alpha \kappa, 1,1), \\
& \alpha^{2} \nu(\alpha \kappa, \alpha \kappa, 1) ; \\
& \alpha^{2} \nu(\alpha \kappa, \alpha \kappa, \alpha \kappa) ;
\end{aligned}
$$

$$
3 \alpha^{2} \kappa \omega+\alpha^{2} \nu=3 \alpha \omega+\alpha^{5} \kappa^{3} \nu
$$

expresses not only a result, but the final result of the action of iodide of methyl on ammonia.

In the case of the chloride of ammonium, $\alpha^{3} \chi^{v}$, putting

$$
a^{3}(1,1,1,1,1,1)
$$

as the symbol of three combined units of hydrogen, we have, effecting the substitution of $\chi$ for 1 and of $\nu$ for 1 ,

$$
\alpha^{3} \chi^{\nu}=\alpha^{3}(\chi, \nu, 1,1,1,1),
$$

in which four substitutions, and no more, of ak for 1 may be effected; whence we have

$$
\alpha^{3}(\chi, \nu, \alpha \kappa, \alpha \kappa, \alpha \kappa, \alpha \kappa)
$$

as the symbol of the unit of the chloride of tetra-methyl-ammonium.

From the point of view of general algebra, $\alpha 1^{2}=\alpha, \alpha^{2} \nu 1^{3}=\alpha^{2} v$, $\alpha^{2} \kappa 1^{4}=\alpha^{2} \kappa, \alpha^{3} \chi^{\nu} 1^{4}=\alpha^{3} \chi^{\nu}$; we are therefore at liberty either to suppress the symbol 1 or to exhibit it according to our convenience. We may compare the symbol $\alpha^{2} v(1,1,1)$ to an open fan, $\alpha^{2} v$ to the same fan folded up. I have said enough to clear up the difficulty of M. Naquet; and this is not the place to further pursue the subject.

With reference to the problem of the expression of "Isomers" on the principles of the Calculus, I will venture to ask M. Naquet and others to suspend their judgment for a time*.

It is a significant fact that a very large proportion of the class of elements which I have termed composite elements have not been found in the sun. In reply to inquiries on my part, Mr. W. Huggins writes to me thus:-

- The following passage and also the footnotes have been added since the publication in the Moniteur Scientifique. 
"So far as I know, nitrogen, phosphorus, arsenic, antimony, boron, chlorine, iodine, bromine, have not been found in the sun. In one paper Lockyer suspects iodine. Dr. Miller and I found coincidence of three lines of antimony with three lines in aldebaran. Though this observation would show considerable probatility of antimony in star, I do not think the spectroscope (two dense prisms of flint glass) was sufficiently powerful to make its existence there certain. In the case of nitrogen, no coincidence was observed in any of the stars. In my paper in the Transactions of the Royal Society on Spectra of Nebulæ, I show coincidence of principal line with the strong line in spectrum of nitrogen. Now this line of nitrogen is a double one; and I was not at first able to be certain if the line in the nebula was similarly double. Subsequently with the powerful spectroscope I used for the motions of stars, I was able to make a certain determination of this point (Proceedings R. S. 1872, p. 385). I found the line in the nebula single and coincident with the middle of the less refrangible of the components of the double line

\begin{tabular}{ll} 
Nitrogen. & Red. \\
\hline & $\|$ \\
\hline Nebula. & -
\end{tabular}

I say "middle" because line in the nebula is narrower and more defined than either of the two lines forming the double line. I made experiments to see if under any conditions of pressure and temperature the more refrangible of the two lines fades out, so as to leave only the one with which the line in the nebula is coincident. I did not succeed. So the matter stands: -Is nitrogen compound? Are there any conditions under which the one line only appears? Has the line in the nebula no real connexion with nitrogen further than being sensibly of the same refrangibility"?

Now we must either consider that the matter of these elements so abundant on the earth does not exist in the sun or stars (which is hardly probable), or that they have passed into forms of combination in which they cannot be recognized by the spectroscope (which is also hardly admissible at that elevated temperature), or that they have been decomposed.

\section{Second Note of M. A. Naquet.}

Sir B. C. Brodie replies victoriously to the principal objection which we brought against him, and begs us to suspend our judgment on the second objection. We will therefore await the new memoir which his last sentence allows us to expect. 


\section{Prof. G. Van der Mensbrugghe on a new Application}

There remain two objections to oppose to Sir B. C. Brodie; but they are of less importance. The first is that Sir B. C. Brodie admits, without any experimental proof, the compound nature of certain bodies considered simple, like chlorine and potassium, while otbers, like mercury, remain simple-although it appears, according to the law of Dulong and Petit, that all our elements ought to be compound, or none of them ought to be compound, at least of those to which the law of Dulong and Petit is applicable. We repeat, however, that though Lockyer's experiences do not conclusively establish the compound nature of our elements, this would be one step towards the verification of Sir B. C. Brodie's hypothesis.

The second objection is that the law of even numbers, which serves as the basis of the new notation, can be thoroughly established in the series of carbon combinations, but it cannot be so completely established in the other series. It would perhaps be simpler to admit that this law is not universal than to admit that chlorine and nitrogen are compound bodies. In any case we cannot do better than repeat, in conclusion, that which we have already said. All hypotheses are but mental artifices to guide us to the discovery of truth; and since a new hypothesis opens new horizons to the investigator, this hypothesis may be fruitful, and ought to be accepted, either by replacing an old hypothesis which has become sterile, or by concurrence with it. It is for this reason that we have translated Sir B. C. Brodie's memoirs; it is for this reason that we do not understand indifference towards his work, and that we should understand such indifference still less now that he has done away with one of the two principal difficulties which we thought we lad found in his system, and that he promises before long to do away with the second.

LXVI. On a new Application of the Potential Energy of Liquid Surfaces. By Professor G. VAN DeR Mensbrugahe*.

TN a recent memoirt I sought to confirm my theory of the 1 variations of potential energy of liquid surfaces by presenting a series of proofs drawn from the observation of liquid films either with two free faces or spread upon another liquid. I had hardly finished the writing of that memoir when my attention was drawn to the remarkable phenomena presented

* Translated from a separate impression communicated by the Author, from the Bulletins de l'AcadémieRoyale de Belgique, $2^{\mathrm{e}}$ série, t. xlvi. no. 11, 1878.

† "Etudes sur les variations d'energie potentielle des surfaces liquides," Mém. de l'Acad. royale de Belgique, 1878, t. xliii. 